\title{
The relationship between psychological health and spiritual wellbeing in Iranian stoma patients
}

\section{Abstract}

Aim: The aim of this study was to investigate the relationship between psychological problems and spiritual wellbeing in Iranian stoma patients. Methods: This descriptive-analytic study was conducted on 70 stoma patients who were enrolled through a convenience sampling method. Depression, anxiety and stress among patients were analysed using Depression, Anxiety and Stress Scale (DASS-21) questionnaire and their spiritual wellbeing was analysed using the Spiritual Wellbeing Scale (SWS). Pearson's correlation test was used to determine the relationship between depression, stress and anxiety with the total spiritual wellbeing and its dimensions. Results: The results of Pearson's correlation test showed that there was an inverse and significant correlation between stress (RR - $0.728 ; P<0.001$ ), anxiety (RR $-0.721 ; P<0.001)$ and depression (RR -0.740 ; $P<0.001)$ with the total score for spiritual wellbeing. Conclusion: These results demonstrated that there is a relationship between psychological problems and spiritual wellbeing in ostomates. That is, patients with higher spiritual wellbeing reported lower levels of psychological problems. The results of the present study can be used for holistic nursing care in these patients.

Hossein Rafiei, Social Determinants of Health Research Centre, Qazvin University of Medical Sciences, Qazvin; Kazem Hosseinzadeh, Social Determinants of Health Research Centre, Qazvin University of Medical Sciences, Qazvin; Mohammad Javad Hoseinabadi-Farahani, University of Social Welfare and Rehabilitation Sciences, Tehran; Ladan Naseh, Ulcer Repair Research Center, Faculty of Nursing and Midwifery, Isfahan University of Medical Sciences, Isfahan; Ali Razaghpoor, Student Research Committee, Qazvin University of Medical Sciences, Qazvin; Sedigheh Aghaei, Isfahan Milad Hospital, Isfahan; Azam Mazroei,

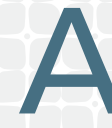
n ileostomy or colostomy is a surgically created exteriorisation of the ileum or colon respectively to the abdominal wall of the patient's body (El-Tawil and Nightingale, 2013). Intestinal stoma formation is a standard treatment for serious bowel conditions. However, patients living with a stoma often face a variety of physical challenges (Box 1) (Nicholas et al, 2008; Sasaki et al, 2017). These can lead to an increase in the prevalence of psychological problems, such as stress, anxiety and depression (White and Hunt, 1997). These pressures are felt by ostomates around the world; in Indonesia 30\% and 45\% respectively reported some degree of anxiety and depression, (Jayarajah et al, 2016), and in Turkey a significant percentage reported psychological problems (Bahayi et al, 2018).

The authors have published two recent articles on caring for stoma patients (Rafiei et al, 2017; 2018). The first article reported the prevalence of psychological problems in stoma patients and the relevant factors driving them. It found a high prevalence of stress ( $87 \%$ ), anxiety (92\%) and depression $(71 \%)$, which was influenced by factors including age, sex, type of stoma and time since operation (Rafiei et al, 2017). The second article studied the relationship between quality of life and spiritual wellbeing in stoma patients. The results showed that a stoma affected every aspect of quality of life, to a degree that in part depended on patients' economic status and time since operation. It also showed a strong positive relationship between quality of life and spiritual wellbeing (Rafiei et al, 2018). Following on from these two studies, a third was proposed to investigate the relationship between psychological disorders and spiritual wellbeing in stoma patients.

Methods

\section{Sample}

The convenience sampling method was used to select 70 participants. This method involves 